\title{
Foundations of the Relative Constancy of Mean Velocity for Natural Streams: A General Principle of Steady State Anticipated by L. Prandtl and L. Leopold
}

\author{
Alfredo José Constaín Aragón \\ I+D+I, FLUVIA, Bogotá, Colombia. \\ E-mail: alfredoconstain@fluvia.co \\ Carlos Peña-Guzmán \\ Environmental and Sanitary Program, \\ La Salle University, Bogotá, Colombia. \\ Corresponding author: cpena@unisalle.edu.co \\ Gina Alexandra Peña-Olarte \\ Environmental Coordination, \\ FLUVIA, Bogotá, Colombia. \\ E-mail: ginapeña@fluvia.co
}

(Received May 4, 2020; Accepted August 1, 2020)

\begin{abstract}
Mean velocity is a key parameter for understanding, modeling and predicting the complex phenomena of advection and dispersion in natural watercourses, which are threatened by human intervention and river disasters. For this reason, the principles of relative constancy for the value of this parameter, established early by L. Prandtl and L. Leopold in the 20th century, are powerful theoretical tools for those who develop engineering tasks in this field. This article presents updated analyses of these principles, explaining how this constancy depends essentially on the existence of a steady state in the flow, which allows equiprobability in the system, and therefore, a minimum production of entropy. At the same time an equation is developed, as a function of macroscopic parameters, facilitating its interpretation and practical application. This hypothesis is contrasted by reviewing the relative values for those parameters and their effect on the formula, and defining the practical conditions of applicability of the formula. This method of validating the hypothesis is important in hydrometric practice, as flow velocity measurements are the starting point for their description, especially for large rivers where comprehensive assessments are impossible.
\end{abstract}

Keywords- Turbulence, Thermodynamics of irreversible processes, Production of entropy, Hydraulic.

\section{Introduction}

Given the great importance today of understanding complex phenomena in natural watercourses, such as advection and dispersion, especially for the characterization, control, and mitigation of their effects in pollution and civil works events, in-depth knowledge of flow velocity behavior and movement patterns is of vital urgency for scientists and engineers researching this field.

Although there are multiple and varied physical magnitudes involved in the formation of flow (Bjerklie et al., 2005; Choo et al., 2014), the mean velocity is the most important, despite the fact that its interaction with the others is a unitary and simultaneous process. The foundational studies on hydraulics by C. Chezy and J, Manning and hydrodynamics by C. Navier and R. Stokes are well known. In these approaches, different aspects of physical reality are presented, such as, in one study, the gravitational component of the tensile force is opposed to the resistant friction force in 
International Journal of Mathematical, Engineering and Management Sciences

Vol. 5, No. 6, 1363-1378, 2020

https://doi.org/10.33889/IJMEMS.2020.5.6.101

the bed of the river to establish a compromise average velocity. In the other study, in a partial differential way, the acceleration of an elementary fluid plot is equal to the sum of forces that push the movement, among which are the internal forces of pressure, tension, and the action external forces. In hydraulics, the mean velocity is immersed in a linear relationship and depends on empirical parameters, and therefore, is easy to handle analytically; in the latter, the point speed is in differential quadratic form, which implies the absence of direct solution methods for its specific value. In general, and due to its own characteristics, the Chezy-Manning semi-empirical equation is commonly applied in river hydraulics engineering situations while the Navier-Stokes partial differential equation is widely used in Fluid Mechanics (Baryshnikov et al., 2011; Graebel, 2007).

These two great dynamic laws of natural watercourses, in reality, cover only a part of the description of turbulent flows (in addition to the continuity equation and Bernoulli's basic law), since the other component of this general behavior corresponds to thermodynamics, which comprises the laws of exchange of energy, mass, and entropy in interacting physical systems (González-Pérez et al., 2019). Both descriptions are indispensable for a rigorous examination of the phenomena that occur in water bodies, and are complementary, each pointing to a series of aspects proper to its field.

As it has been generally said, velocity is perhaps the most important physical magnitude of the river phenomenon, since it is directly associated with advection, and since, in essence, this concept is defined precisely as the movement of water flow. Also, apart from the turbulence itself, dispersion occurs, which understood as the scattering of the particles of a solute in the flow thanks to the mutual distancing of any random pair of these particles, fundamentally due to the shear effect of the flow and aided by the physical-chemical forces of reaction to the disturbance of chemical equilibrium (Le Chatelier-Braun effect). The shear effect is due to the presence of random velocity vectors that impart different impulses to the particles and are derived precisely from the presence of the mean speed. For that reason, dispersion depends ultimately on the mean velocity (Fischer, 1967; Seo and Cheong, 1998).

This article analyses in detail the different aspects of the nature of average flow velocity and its relationship with its applications to fluvial engineering. It covers both the dynamic part itself and thermodynamics. A central aspect in this analysis is that of the relative constancy of the value of the average speed in a given stretch of river. The qualification relative means that the mean velocity is not a bare number but a random variable, with an average value and a probabilistic distribution of fluctuations (variations).

\section{Discussion}

\subsection{Complex Nature of Flow Velocity: Current Lines and Turbulence}

The movement of water in a natural channel is described by a continuous vector field of velocities, defined for a given point and time. A practical way of looking at the set of vectors of such a field is through the concept of flow lines, which are geometric places at each point and time where the vector is tangent to the line.

The set of current lines in a specific plot of the flow is called the "current tube", which is the geometric location of that specific vector field, and a higher density of lines indicates vectors with larger moduli. If the lines are very close together there is a higher modulus, if they are separated the modulus is lower. 
International Journal of Mathematical, Engineering and Management Sciences

Vol. 5, No. 6, 1363-1378, 2020

https://doi.org/10.33889/IJMEMS.2020.5.6.101

A current tube in a natural channel subject to the action of turbulence is an intuitive, useful, but very thick representation (coarse-grained) because the actual movements of the flow filaments are too complex, heterogeneous, and variable to be represented in their most intimate essence, and the flow lines must be understood as representations of averages. Depending on the velocity, natural flow changes from laminar to turbulent. Turbulence, a disordered rotational movement in all directions, is superimposed on the ordered advective movement of the flow. Turbulent rotations and displacements are very entangled, chaotic movements, but they can involve a very large number of particles. From the point of view of the kinetic energy frequency spectrum of these chaotic movements (Fourier distributions) the passage to turbulence implies a great widening of that band.

\subsection{Phenomena That Depend on the Flow Velocity}

\subsubsection{Turbulence and Average Flow Velocity}

If the average flow velocity is assigned a predominant position, it is necessary to make a basic count of the processes that depend on this parameter. It is convenient to start with the turbulent movement. As already mentioned, turbulence is a chaotic state of the fluid in motion that gradually develops from laminar motion, in which viscous forces maintain orderly movement, until kinetic (inertial) forces release that force-jacket. To estimate this commitment relationship between two types of forces, the velocity dependence of the Reynolds' Dimensionless Number is defined as:

$\operatorname{Re} \approx \frac{U * L}{v}$

Here, $U$ is the average velocity of the flowing substance, $L$ is a characteristic length of the system, and $v$ is the kinematic viscosity of the liquid (water).

\subsubsection{The Process of Dispersion in Turbulent Flows}

As already explained, the dispersive dynamics of substances dissolved in the flow, which consists of the random separation of any pair of particles, depend fundamentally on the speed. Therefore, the dispersive $(E)$ and diffusive transport coefficients $(\varepsilon y)$, which lead to the dilution (decrease of the peak concentration) and scattering (spread) of the substance, are established according to this parameter.

$$
E, \varepsilon \boldsymbol{y} \sim \boldsymbol{f}(\boldsymbol{U})
$$

\subsection{The Relative Constancy of Average Velocity. Background According to Leopold from a Statistical Study in the 1950s}

\subsubsection{Basis for the Study of Velocity Behavior in a Series of Watercourse}

One of the first and most significant studies in contemporary technical literature is the one carried out by Leopold (Leopold, 1953); he analyzes in detail extensive statistics of flow and average velocity measurements through a network of hydrometric stations in the United States. The objective of the study was to characterize the change in mean velocity downstream in the rivers considered. From the number of streams considered, it is clear that there must be some statistical dispersion, decreasing, conserving or increasing the downstream velocity, from one case to another, but the focus is on the average trend.

The flow was measured with a current meter, or with curves calibrated with the level data. The 
International Journal of Mathematical, Engineering and Management Sciences

Vol. 5, No. 6, 1363-1378, 2020

https://doi.org/10.33889/IJMEMS.2020.5.6.101

average speed was obtained using the continuity relationship:

$U \approx \frac{Q}{A_{Y Z}}$

Where, $A_{y z}$ is the cross-sectional area, supposedly quite stable and congruent with a prismatic shape. The advantage of this study is that, even if one were to think in the absence of models that anticipate statistical constancy, its results can give reliable insights into the behavior of this parameter over time and distance.

Leopold analyzed a universe of about 6000 natural streams of diverse nature and magnitude measured by the USGS during a rather extensive period from 1923 to 1950; to make them comparable, it was necessary to establish an equivalence criterion so that the flow was intrinsically representative according to hydrological practice. For this purpose, the flow data series were taken over time and curves were made in which the flow durations and maximum flow frequencies and their recurrence times were characterized. Then the average velocity measurements in the same channel were made when the conditions were equivalent in the duration and frequency curves, for the stations involved. The representative calculation value in these curves was the one corresponding to the average annual flow.

For the specific practical purposes of the study, only some of the most representative channels in the series were chosen, e.g. the Yellowstone, Missouri and Mississippi watersheds.

\subsection{Analysis of Prandtl's Lateral Longitudinal Velocity Distribution}

An important aspect of river dynamics is to know the lateral (Y-axis) distribution of the longitudinal flow velocities, i.e. across the width of the stream. This distribution has been found to regularly accommodate stream tube flow lines, evenly spaced except for a bias towards the edges.

The theoretical work of approximating this distribution in natural flows was mainly undertaken by Prandtl. His approach was to apply the effect of viscosity as a delaying agent in the movement of the fluid towards the edges. From his considerations, the modern general version of this distribution can be written as follows:

$\frac{U L}{U m x m}=a * \operatorname{Ln}\left(b * \frac{W L}{\frac{W}{2}}+c\right)+d * \frac{W L}{\frac{W}{2}}+e$

Where, $U L$ is the local velocity at an arbitrary distance given from the channel edge to the center of the flow, $U$ max is the maximum flow velocity at the center of the channel, $W L$ is the local lateral distance where the $U L$ measurement is made, and $W / 2$ is the lateral distance to the center of the channel. Note that due to the symmetry of the distribution, only what occurs from an edge to the center of the flow is represented; the total width of the flow is, therefore, $W$.

According to Prandtl's approach, the bias of its distribution corresponds to the effect of viscosity, which is more predominant as the point considered gets closer to the edge of the channel. Since the correction for this bias effect is logarithmic, and this is the shape of that bias in the curve, it can be stated that, if there were not this distorting effect, the distribution itself would be a straight line, i.e. all velocities with the longitudinal direction have the same average probability of occurring 
International Journal of Mathematical, Engineering and Management Sciences

Vol. 5, No. 6, 1363-1378, 2020

https://doi.org/10.33889/IJMEMS.2020.5.6.101

regardless of the transverse position.

\subsection{River Dynamics: State of Equilibrium and The Factors That Modify it}

The states of equilibrium in natural riverbeds have been studied for a long time (Leopold and Langbein, 1962), equilibrium being understood as the permanence in time of the geometric characteristics or of the geomorphological ones. This implies invariance in time for its transversal and longitudinal profile, as well as for its plan route.

The stability of natural flow is usually a long-term concept; that is, they are usually slow events. For this reason, strictly speaking, the parameters that determine it, such as flow rate or slope, for example, are calculated using statistical models from mathematical hydrology, which involve as much information as possible in terms of distances, frequencies, maximums, minimums, and multiplicity of structures, etc.

The equilibrium of a channel, on the other hand, is not an absolute concept, but rather relative; that is, it has a statistical, or dynamic sense. Fluctuations in one direction of the factors that determine it occur with the same average frequency in the other direction. The factors that determine the equilibrium are all interconnected. For example, the liquid flow (which transports pure fluid) is in equilibrium with the solid flow (which transports substances in suspension or dissolved) in such a way that hydraulics interferes with geomorphology and vice versa. The transport involved in the solid flow has a great influence on the criteria to define stability in the channels.

It is necessary to have a primary guide to the interpretation of the change observed in the data of the same river; therefore, the Chezy-Manning equation is proposed as such a reference.

$U \approx \frac{R^{\frac{2}{3}}}{n} * \sqrt{S}$

A practical way to estimate the deviation of a natural channel from the steady state condition is to measure the longitudinal gradients of hydraulic radius, roughness, and Slope. As long as those gradients are very small (tending to zero), the measured stretch will be reasonably in that condition.

\subsubsection{Processes of Searching for Dynamic Equilibrium in Watercourses}

Natural watercourses are systems that are permanently adjusting, or self-regulating in time and space, producing equilibrium in their dynamics. In general, equilibrium occurs in physical systems when there are opposing forces that neutralize each other. This is the most common general scheme, which prevents such systems from avalanches or runaways when equilibrium is broken, and one effect predominates over the other; this sometimes happens but fortunately, it is not the most frequent. Equilibrium is established when the opposite effects are tuned in to even the smallest details, and statistical or dynamic harmony is born. A first equilibrium can be defined when erosion shapes, either by deepening or widening the width of flow until a compromise between the two opposing effects is achieved (see Figure 1).

The second type of equilibrium corresponds to the mutual adjustment of erosion and deposition of bed material. This occurs because the mass transport mechanism in the flows either leaves or removes material at the same rate, given the equivalence of the mechanisms associated with each effect. 
International Journal of Mathematical, Engineering and Management Sciences

Vol. 5, No. 6, 1363-1378, 2020

https://doi.org/10.33889/IJMEMS.2020.5.6.101
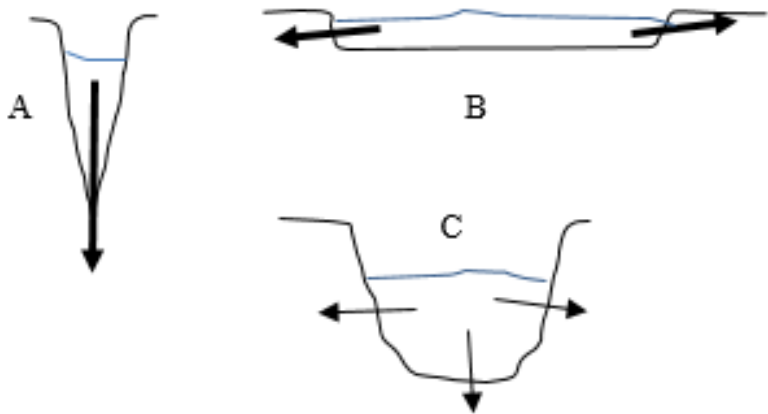

Figure 1. Mutual control of depth and width in a natural flow. First equilibrium (A), second equilibrium (B) and third equilibrium $(\mathrm{C})$

The third process of adjustment has to do with the factors involved in the Chezy-Manning equation, to achieve a net balance between dynamic effect and resistance; then, if the changes in geomorphology make it so there is a tendency to increase the average speed (increase $R$ or decrease $n$ ), the flow will look for ways to diminish the slope, by creating meanders.

This continuous and paused process of adjusting the slope of a riverbed is done by interspersing high and low-speed passes which will make the watercourse more uniform as its development advances, from rocky channel to alluvial channel, and the flow course itself will seek the appropriate meandering route to achieve this.

In summary, the dynamic function of natural flow is to transport its liquid and solid flows, in which it invests a certain amount of energy, from its reservoir supplied by the gravitational component. The rest of that energy is used to modify the geomorphology of the path so that it accommodates the search for balance, with great thermodynamic significance, as will be seen below.

\subsection{Thermodynamics of the Irreversible Processes}

To begin with, thermodynamics, as opposed to dynamics, has been characterized as a branch of science that does not start from any structural model of substance in which the balances of energy, entropy, and substance that are counted at the boundaries of the systems are fundamentally important (Bejan, 2016; Zivieri et al., 2017). It is, therefore, a science with a large dose of certainty and credibility since it is strongly anchored in experimental results to feed its developments. It is also a scientific methodology that can potentially provide information from larger plots, different from the local measurement point, under certain conditions that will be analyzed.

Its method complements quite well with the so-called Statistical Mechanics, a science that is essentially theoretical. Its subject of study is the so-called probability density distributions, which are numerical characteristics of the random variables, which are mathematical constructs associated with entropy, fundamentally.

Although, Thermodynamics was born at the dawn of the modern era, as a necessity to understand the essence of heat and its transformations, essentially describing processes in equilibrium, that is, reversible processes; its scope has been extended to phenomena outside the equilibrium, that is, irreversible processes, which are the vast majority of what occurs in the real world (Kondepudi and 
International Journal of Mathematical, Engineering and Management Sciences

Vol. 5, No. 6, 1363-1378, 2020

https://doi.org/10.33889/IJMEMS.2020.5.6.101

Prigogine, 2014).

The direct way of describing irreversibility has been to consider real open systems that exchange energy and substance at their boundaries, and in which there is an exchange of reversible entropy (ideal) at the border, $d e S$, due to the crossing of reversible heat (unrelated to temperature gradients) or substance, and internal production of entropy by loss effects, diS. In isolated systems:

$\operatorname{deS}=\mathbf{0}$

In open systems in non-thermodynamic equilibrium, both terms make up the total change of entropy in the system, $d S$ :

$d S=d i S+d e S$

While diS is always positive, deS can be positive or negative, depending on whether the reversible heat goes in or out:

$d e S= \pm \frac{d Q}{T}$

In reversible systems:

$\operatorname{diS}=\mathbf{0}$

\subsubsection{The Stationary State in the Irreversible Processes: System Stability}

For an open system, in 1945 Prigogine established the Theorem of "Minimum entropy production" for the condition of "stability" or steady state, which does not change with time in a relative, statistical sense. This theorem shows that in this condition, the entropy is a maximum and the production of entropy, $\sigma=d i S / d t$, is a minimum. For the maximum entropy, its differential is zero, $d S=0$, and it is true that:

$d S=d i S+d e S=0$

If the internally generated entropy is equal to the entropy expelled at the border in absolute value, the minus sign indicates that the entropy leaves the system:

$\operatorname{diS}=-\operatorname{deS}$

An important parameter at this point is the so-called entropy production, defined for the unit of volume of a system, $\sigma$, where the local equilibrium is fulfilled, which allows accepting that entropy is well defined for irreversible processes.

$\sigma=\frac{\text { dis }(\text { unitary volume })}{d t}$

For the minimum entropy production, the loss energy, which is the propitious one to generate increasing fluctuations, must be expelled to the external environment. This ensures that these fluctuations are damped, and the system is therefore stable (Varadhan, 2015). 
International Journal of Mathematical, Engineering and Management Sciences

Vol. 5, No. 6, 1363-1378, 2020

https://doi.org/10.33889/IJMEMS.2020.5.6.101

\subsubsection{Stationary State in the Irreversible Processes: Predominance of the Linearity Between Forces and Flows}

If it is assumed that for a system outside the equilibrium there exists at least a local equilibrium in a unit volume, which the thermodynamic variables of each subsystem of that system properly divided, admit the same interpretation as in the equilibrium, it can be shown that for such a system that is stable (with minimum entropy production) the thermodynamic forces, $F$, generate flows, $J$, proportional to each other; that is to say they have linear dynamics (Bejan, 2016). Then, for a unit volume system:

$\sigma=\frac{d i s}{d t} \rightarrow$ Minimum in Local Equilibrium

Consequently $\boldsymbol{F} \sim \boldsymbol{J}$. In summary, irreversible systems in which thermodynamic forces and flows are proportional and fluctuations are damped by having their entropy output at a minimum are stable.

\subsection{Steady State in Watercourse. Statistical Constancy of Velocity as a Consequence of the Minimum Entropy Theorem and Boltzmann's Definition}

Stability in natural streams has been a recurrent topic of study in hydraulics for more than a century. However, the studies that L. Leopold and others from the USGS in the United States carried out by investigating a large number of streams in that country are classics (Leopold et al., 1964).

The main axis of these studies has been the theorem of minimum entropy production and maximum entropy. This last one, put in the terms of L. Boltzmann's definition, allows us to establish that all the $n$ micro-events are equivalent, that is, ergodic, and therefore their probabilities will be the same.

If this condition of equiprobability is applied to energy events, it can be shown that the particles in the flow, when passing from one position to another, retain their mass transfer rate, and therefore, their velocity, thus showing a constant average speed at any point in the flow. From this, the two principles of Leopold and Prandtl are derived:

A. Leopold: "The velocity (longitudinal) of a riverbed, in the longitudinal sense, is sensibly similar in the high part and in the delta"

B. Prandtl: "The longitudinal speed of a riverbed in the transversal direction, is sensibly similar to the width, except for the retarding effect of the viscosity at the edges.

Therefore, natural flow in stability conditions will show a statistical constancy of the average speed, seen both in it's longitudinal and transversal dimensions due to thermodynamic reasons.

\subsubsection{Is the Turbulence Stable and up to What Limits? Its Dependence on the Average Velocity. An Analogy between Dispersion and Turbulence}

If we start from the chaotic interpretation of turbulence, in which the fluctuations are amplified by non-linear mechanisms, the question is whether this process of heterogeneous mixing, in which the rotational movements are degraded on decreasing Kolmogorov scales, has a limit at which it stabilizes, i.e. does not increase until a run-off occurs.

An important point in this respect is to see how the degree of instability of the turbulent flow, size, 
International Journal of Mathematical, Engineering and Management Sciences

Vol. 5, No. 6, 1363-1378, 2020

https://doi.org/10.33889/IJMEMS.2020.5.6.101

and distribution of the velocity fluctuations, is described as a function of the Reynolds Number, and this, in turn, is described as a function of the average speed. It is clear that the instability of the turbulent system depends critically on the value of the average speed, and therefore, it can be said that a turbulent system is stable when the velocity is constant. In this case, the Reynolds Number has a fixed value, and therefore, the level of fluctuations can also be maintained at a fixed value without leading to runaway processes.

Taking into account what is expressed in 2.6.1, an irreversible thermodynamic system is stable when the thermodynamic forces and flows are proportional. Here the analogy between turbulence and scattering is useful to detail it and to draw some conclusions regarding the stability of the first one.

Scattering occurs when particles in a tracer are mixed and spread in a turbulent flow, mainly by a shear effect. Now, on the basis that these particles and those of the water are very similar and subject to the same laws, the tracers are faithful witnesses to the movements of the water particles, and therefore, the particles of the fluid disperse in turbulence. The difference is that while the tracer corresponds to a finite injection of mass, and therefore relaxation is observed until that mass differential is finally balanced, the mass of the water flow is constantly fed and no relaxation is identified. If this is so, and if the system is stable, the flow and thermodynamic force are proportional in the dispersion:

$J \sim D \frac{\partial C}{\partial X}$

Similarly, in turbulent flow, proportional flow and force can be identified. Likewise, for this type of turbulent movement, entropy production can be defined, and this expression should tend to a constant, small value within the corresponding approximations that guarantee the extinction of speed fluctuations in the turbulence.

\subsection{Approximate Determination of the Entropy Production Function, $\Sigma$, in Turbulence and Velocity Behavior in Watercourse}

It is clear from this article that there are sufficient reasons of various kinds to consider that under normal or stable conditions of a riverbed, speed will be a consistently constant parameter. A quantitative estimator to support the above statement is to calculate the production of entropy for turbulence, which as we have already seen, may have similar characteristics to those of dispersion and may indicate that turbulence behaves linearly from the point of view of the thermodynamics of irreversible processes.

\subsubsection{Development of the Basic Formula for $\sigma$}

The definition of entropy production for the specific case of a turbulent flow is assumed, taking into account that entropy is conserved, Equations (6) and (7), and that the exchange entropy is defined as a function of heat, $d Q$, represented in frictional losses on the water to ground interface and shear losses transferred in the process, assuming an isothermal condition to ignore other contributions of heat transfer with the environment by thermal gradients, and assuming that there are no other irreversible losses.

$d i s \approx d s e \approx \frac{d Q}{T}$ 
International Journal of Mathematical, Engineering and Management Sciences

Vol. 5, No. 6, 1363-1378, 2020

https://doi.org/10.33889/IJMEMS.2020.5.6.101

Therefore, if $\frac{d i S}{d t}=\frac{d e S}{d t t}$ and taking into account the definition of potential energy as a function of height, $h$, and the mechanical equivalent of heat, one has

dis $\approx \frac{4.184 * k 2 * d(m * g * h)}{T}$

Now, by defining the potential energy change as a function only of height, and calculating the production of internal entropy, $\sigma$, one has

$\sigma \approx \frac{d i s}{d t} \approx \frac{4.184 * k 2 * m * g * d h}{T * d t}$

If this is multiplied and divided by the differential of distance, $d X$, if it is assumed that the mass is transferred by lots equal to $d m$, and if the equation is reorganized, taking into account that the inverse of the temperature is convenient for calculating the gradient, one obtains

$\sigma \approx 4.184 * k 2 * g * d X * \frac{d m}{d t} * \frac{1}{T} \frac{d h}{d X}$

If this is multiplied and divided by $A y z$, i.e., the area of the cross section of the flow, and knowing that the acceleration due to gravity, $g=9.81\left(\mathrm{~m} / \mathrm{s}^{2}\right)$, one obtains

$$
\sigma \approx 41.1 * k 2 * A y z * d X * \frac{\left(\frac{d m}{A y z}\right)}{d t} * \frac{1}{T} \frac{d h}{d X}
$$

Now if we take into account that the product of $A y z$ by $d X$ is the volume of the leg, $V$, we have finally expression for general entropy production (for the whole volume):

$\sigma^{\prime} \approx 41.1 * k 2 * V * \frac{\left(\frac{d m}{A y z}\right)}{d t} * \frac{1}{T}\left(\frac{d h}{d X}\right)$

In the previous expression we have that the temporal derivative is the mass transport rate in the flow, $T_{t m}$, and the spatial derivative is the slope (Constaín et al., 2019a). Now, it is important to interpret the above expression in terms of thermodynamic forces and flows. Thus, the force is the spatial gradient affected by the inverse temperature, and the flow is the temporal derivative:

$F \sim \frac{1}{T}\left(\frac{d h}{d X}\right)$

$J \sim\left(\frac{d m}{d t}\right)$

Now, under the condition that the irreversible process occurs near thermodynamic equilibrium, and therefore is linear, the flow has to be proportional to the force:

$\left(\frac{\frac{d m}{A y z}}{d t}\right) \approx L * \frac{1}{T}\left(\frac{d h}{d X}\right)$ 
International Journal of Mathematical, Engineering and Management Sciences

Vol. 5, No. 6, 1363-1378, 2020

https://doi.org/10.33889/IJMEMS.2020.5.6.101

Therefore, taking into account the above equations, and remembering that the height gradient is precisely the slope, Equation (20) becomes

$\sigma^{\prime} \approx \frac{41.1}{T^{2}} * k 2 * V * L * S^{2}$

It is necessary to find a unified value for the factors $L$ and $k 2$. So, $L$ : From Equation (23) this constant can be calculated as

$L \approx \frac{T *\left(\frac{d m}{A y z * d t}\right)}{S}$

Bearing in mind that the mass differential can be written as a function of the density of the water, $\sigma$, and the volume differential, and that this differential divided by the cross-sectional area is $d X$, one has

$L \approx \frac{T *\left(\frac{\rho * d V}{A y z * d t}\right)}{S} \approx \frac{T}{S} * \frac{\rho * d X}{d t} \approx \frac{T * \rho * U}{S}$

$\boldsymbol{k} 2$ : The energy that enters the open system is that which generates the movement of the flow according to the decrease of the slope, that is to say the potential energy, which is then transformed into a kinetic part delimiting the work associated with the movement of the flow, and the generation of heat by action of the frictional forces that are transmitted to the environment across the border. Using the first law of thermodynamics, one has

$d U=d A+d Q$

For the purposes of further calculation, it is convenient to define the working differential and heat as fractions of the input potential energy:

$d A=k 1 d U$

and

$d Q=a k 2 d U$

Here, $a \approx 4.184$ (joules/calorie) is the mechanical equivalent of heat, since the heat differential will be replaced by mechanical units of energy, of course $k 1+k 2=1$.From Equation (29) one has

$k 2 \approx \frac{\frac{1}{4.184} * d Q}{d U} \approx 0.239 * \frac{d Q}{d U}$

Now, according to Bernoulli's equation, the energy derivative can be expressed as a function of the height

$\frac{d Q}{d U} \quad \frac{h_{\text {loss }}}{h}$ 
International Journal of Mathematical, Engineering and Management Sciences

Vol. 5, No. 6, 1363-1378, 2020

https://doi.org/10.33889/IJMEMS.2020.5.6.101

The classic definition of height for turbulent losses for a rough tube roughly assimilated to an open channel, such as in, is

$$
h_{\text {loss }} f * \frac{L * U^{2}}{(4 * R) * 2 * g}
$$

Where, $R$ is the hydraulic radius, $U$ is the average velocity of the flow, and $f$ is the turbulent loss coefficient of Darcy-Weisbach, which is approximately

$f \approx \frac{8 * g}{C^{2}}$

with $C$ being Chezy's coefficient, and also as a function of Manning's roughness, $n$ :

$f \approx \frac{8 * g * n^{2}}{R^{\frac{1}{3}}}$

Therefore, the parameter $k 2$ becomes

$k 2 \approx 0.239 * \frac{L *(n * U)^{2}}{h * R^{\frac{4}{3}}}$

Now, with $L / h \approx(1 / S)$ :

$k 2 \approx 0.239 * \frac{(n * U)^{2}}{S * R^{\frac{4}{3}}}$

Therefore, the product of these two parameters is

$L * k 2 \approx 0.239 * \frac{T * \rho * U^{3} * n^{2}}{S * R^{\frac{4}{3}}}$

Combining Equations (24) and (37) and simplifying, the production of entropy for turbulent flows becomes, approximately,

$\sigma^{\prime} \approx \frac{9.82}{T} *\left(\frac{\rho * U^{3} * n^{2} * S}{R^{\frac{4}{3}}}\right) * V$

where, $T$ is the absolute temperature, $\rho$ is the water density, $U$ is the average velocity in the considered sub-section, and $V$ is the liquid volume of the considered sub-section.

And that the density of entropy is therefore

$\sigma \approx \frac{9.82}{T} *\left(\frac{\rho * U^{3} * n^{2} * S}{R^{\frac{4}{3}}}\right)$

If we take into account that for usual values of the ranges of macroscopic parameters, we can verify 
International Journal of Mathematical, Engineering and Management Sciences

Vol. 5, No. 6, 1363-1378, 2020

https://doi.org/10.33889/IJMEMS.2020.5.6.101

that the value of $\sigma$ is small in absolute value, and that its change is equally small, if the differentials $d n, d R$ and $d S$, are small, i.e. approximately fulfilling the requirement that the thermodynamic force and flow implied by Equation (39) are proportional and that the equiprobability condition leads to the consideration of flow velocities throughout the turbulent system as converging.

This definition is obtained by taking several reasonable approaches, namely that the system does not contemplate chemical reactions that affect the energy and entropy balances, that it remains isothermal within and in relation to its environment, eliminating internal and external transfers of irreversible heat, that there is a mass balance between that which enters (by sediments) and that which leaves (by erosions), which avoids having to consider net entropies associated with this transfer and also justifies the condition of stable state for the system by maintaining an optimal and constant cross-section, and that sources of irreversibility other than friction and shear losses are not taken into account; that is, additional phenomena that change the state in finite times are avoided.

\subsubsection{Conditions of Application of the Theory}

To approximately calculate definition (39), which depends on geomorphological, geometric, and dynamic parameters of direct measurement and interpretation, it is convenient first to define the extension of the section, in which if it is relatively short, such that the values of the variations in velocity, slope, hydraulic radius and roughness therefore $\sigma$ as constant as possible, given that their absolute value is a small value, since the following product tends to zero, according to previous study by authors (Constaín et al., 2019b). And also:

$$
\frac{\left(n^{2} * S\right)}{T} \rightarrow \mathbf{0}
$$

In this way, $\sigma$ is maintained at a minimum value, complying with Prigogine's Theorem of Minimum entropy production in the linear region of irreversible thermodynamics, and allowing the dissemination of equiprobability throughout the flow environment, and therefore ensuring a condition of maintenance of the value of speed, as Prandtl and Leopold predicted.

\subsubsection{Practical Application of the Concepts Developed}

In order to put into approximate numerical perspective the concepts exposed in this Article, an experiment with tracers is documented. This experiment was carried out in the Ure River, in the Northeast of Colombia, a relatively large riverbed of 30 meters wide and a flow of approximately $200 \mathrm{~m}^{3} / \mathrm{s}$, whose data was taken in the field by the technical team of the company FLUVIA TECHNOLOGIES with its equipment for measuring tracers in real time.

Table 1. Data on experimental parameters in the Rio Ure section

\begin{tabular}{|l|c|c|c|c|c|c|}
\hline Measurement point & Distance $(\mathrm{m})$ & Width $(\mathrm{m})$ & Hydraulic radius $(\mathrm{m})$ & Slope $(\mathrm{m} / \mathrm{m})$ & Roughness & Velocity $(\mathrm{m} / \mathrm{s})$ \\
\cline { 1 - 6 } A ( Source ) & & & & & & 0.0001 \\
\hline B Injection 1 & 3900 & 30.0 & 4.29 & 0.000 & 0.95 \\
\hline C Injection 1 & 5184 & 35.0 & 4.29 & 0.0002 & 0.035 & 0.035 \\
\hline D Injection 2 & 9650 & 30.0 & 3.26 & 0.0003 & 0.035 & 0.10 \\
\hline E Injection 2 & 14116.7 & 30.5 & 3.17 & 0.0002 & 0.035 & 0.90 \\
\hline Mean & --------- & 31.4 & 3.80 & 0.0002 & 0.035 & 0.96 \\
\hline
\end{tabular}

First tracer injection (Rhodamine WT) is made at point A $(0 \mathrm{~m})$ and the tracer is measured 
International Journal of Mathematical, Engineering and Management Sciences

Vol. 5, No. 6, 1363-1378, 2020

https://doi.org/10.33889/IJMEMS.2020.5.6.101

sequentially at points B (Station 1 at $3900 \mathrm{~m}$ ) and C (Station 2 at $5184 \mathrm{~m}$ ), then with reference to point $\mathrm{C}$, a second tracer injection is made, which is measured sequentially at points $\mathrm{D}$ (Station 1 at $9500 \mathrm{~m}$ with respect to A) and $\mathrm{E}$ (Station 2 at $14116.7 \mathrm{~m}$ with respect to A). According to the measurements and observations, we have the following Table 1 of average data of the experiment.

Now, on the data of the Table 1, it is possible to make an estimate of the variations in absolute value, in relation to the distance. See values in Table 2

Table 2. Table of differences in parameters as a function of distance

\begin{tabular}{|l|c|c|c|c|c|}
\hline \multicolumn{1}{|c|}{ Estation } & $\Delta$ Distance $(\mathrm{m})$ & $\begin{array}{c}\Delta \text { Hydraulic } \\
\text { radius }(\mathrm{m})\end{array}$ & $\Delta$ Slope & $\Delta$ Roughness & $\Delta$ Velocity $(\mathrm{m} / \mathrm{s})$ \\
\hline B & $\Delta \mathrm{X} 1=1284$ & $\mathrm{AR} 1=0.0$ & $\Delta \mathrm{S} 1=0.0001$ & $\Delta \mathrm{n} 1=0.0$ & $\Delta \mathrm{U} 1=0.03$ \\
\hline C & $\Delta \mathrm{X} 2=4466.7$ & $\Delta \mathrm{R} 2=0.09$ & $\Delta \mathrm{S} 2=0.0001$ & $\Delta \mathrm{n} 2=0.0$ & $\Delta \mathrm{U} 2=0.20$ \\
\hline
\end{tabular}

A first calculation with the data of Table 1 , is the value of entropy production per unit volume for the turbulent channel under study, with $T \approx 300 \mathrm{~K}, \rho\left(\mathrm{H} 2 \mathrm{O}\right.$ at $\left.4^{\circ} \mathrm{C}\right) \approx 1000 \mathrm{~kg} / \mathrm{m}^{3}, U \approx 0.96 \mathrm{~m} / \mathrm{s}$, $n \approx 0.035, R \approx 3.80 \mathrm{~m}$, and $S \approx 0.0002$, is available:

$\sigma \approx \frac{9.82}{T} *\left(\frac{\rho * U^{3} * n^{2} * S}{R^{\frac{4}{3}}}\right) \approx 0.033 * \frac{1000 * 0.922 * 0.00123 * 0.0002}{5.93} \approx 1.2 \times 10^{-6} \frac{\mathrm{J}}{\mathrm{Km}^{3}}$

And its variation in the distances considered, for the calculation of $\Delta \sigma$, the absolute increments calculated in Table 2 are added to the absolute values.

$\sigma+\Delta \sigma \approx \frac{9.82}{T} *\left(\frac{\rho *(U+0.2)^{3} * n^{2} * S+\Delta S}{R+0.09^{3}}\right) \approx 0.033 * \frac{1000 * 0.922 * 0.00123 * 0.0002}{6.12} \approx 8.8 x 10^{-5} \frac{\mathrm{J}}{\mathrm{Km}^{3}}$

It can be seen that it is a very small absolute value, and its variation in equal distance is negligible, compatible with the Prigogine Theorem, justifying the assumption of equiprobability for turbulent flow. The rate of change of the parameters of the Chezy-Manning equation can be see in Table 3 .

Table 3. Rate of change of the parameters of the Chezy-Manning

\begin{tabular}{|c|c|c|c|}
\hline$\frac{\Delta U}{\Delta X}$ & $\frac{\Delta n}{\Delta X}$ & $\frac{\Delta R}{\Delta X}$ & $\frac{\Delta S}{\Delta X}$ \\
\hline$\frac{0.20 \frac{m}{s}}{4466.7 \mathrm{~m}} \approx 2.24 * 10^{-8}$ & 0 & $\frac{0.09 \mathrm{~m}}{4466.7 \mathrm{~m}} \approx 2.01 * 10^{-5}$ & $\frac{0.20 \frac{\mathrm{m}}{S}}{4466.7 \mathrm{~m}} \approx 4.47 * 10^{-5}$ \\
\hline
\end{tabular}

It is therefore verified that the variation of the parameters with the distance, for this case is minimal, indicating that the steady state for the riverbed is satisfactorily fulfilled. 
International Journal of Mathematical, Engineering and Management Sciences

Vol. 5, No. 6, 1363-1378, 2020

https://doi.org/10.33889/IJMEMS.2020.5.6.101

\section{Conclusions}

In this article, the principles of Leopold (longitudinal constancy of mean velocity) and Prandtl (transverse constancy of mean velocity) are used as hypotheses to justify the thermodynamics of irreversible processes. The method of verification of the hypothesis is to justify that the production of entropy, $\sigma$, tends to zero and is relatively constant over not very long stretches, in which the variation of geomorphology is insignificant, and that therefore the consequent Boltzmann equiprobability is omnipresent, which implies convergent velocities at all points.

Applying the thermodynamic developments of irreversible processes and proposing the ideal entropy balance in a section of the river, considered as an open system, it is feasible to determine an approximate formula for its entropy production.

Unlike the usual approaches to address this issue, which are based on a punctual (differential) analysis and whose integration is usually plagued by great analytical difficulties, this article uses an integral (non-local) approach, allowing us to obtain an expression based on macroscopic variables, with a direct meaning for the experimenter.

The expression obtained, of a practical nature, allows us to infer that for non-long stretches, the production of entropy in natural streams remains relatively constant and of small value, thanks to the presence in the numerator of normally very small magnitudes, such as the slope and the roughness squared.

This condition allows us to reasonably assume that the equiprobability condition is already reached in the flows in normal condition (steady state) and that therefore the velocity is a relatively constant parameter in the sense of Leopold and Prandtl.

\section{Conflicts of Interest}

The authors confirm that there is no conflict of interest to declare for this publication.

\section{Acknowledgement}

The authors thank the anonymous reviewers for strengthening and contributing to the improvement of the article.

\section{References}

Baryshnikov, N., Pagin, A., Demidova, Y., \& Selina, T. (2011). On the influence of the features of morphologic structure of the floodplain and riverbed on the hydraulics of streams in channels with floodplains. Russian Meteorology and Hydrology, 36(12), 826-829.

Bejan, A. (2016). Advanced engineering thermodynamics. John Wiley \& Sons, Hoboken, New Jersey.

Bjerklie, D., Dingman, S., \& Bolster, C. (2005). Comparison of constitutive flow resistance equations based on the Manning and Chezy equations applied to natural rivers. Water Resources Research, 41(11). https://doi.org/10.1029/2004WR003776.

Choo, T., Chae, S., Yoon, H., \& Choo, Y. (2014). Discharge prediction using hydraulic characteristics of mean velocity equation. Environmental Earth Sciences, 71(2), 675-683. 
International Journal of Mathematical, Engineering and Management Sciences

Vol. 5, No. 6, 1363-1378, 2020

https://doi.org/10.33889/IJMEMS.2020.5.6.101

Constaín, A., Peña-Guzmán, C., \& Mesa, D. (2019a). Determination of hydraulic features in Colombian rivers by tracer analysis. International Journal of Hydrology Science and Technology, 9(5), 457-473.

Constaín, A., Peña-Olarte, G., \& Peña-Guzmán, C. (2019b). Determination of the factors of variation of mean velocity in natural channels at steady state. DYNA, 86(210), 240-246.

Fischer, H. (1967). The mechanics of dispersion in natural streams. Journal of the Hydraulics Division, 93(6), 187-216.

González-Pérez, M., Jalomo-Aguirre, F., \& Lizcano-Caro, J. (2019). Water resources in urban systems: El Zapotillo dam as occasional negentropy in the metropolis of Guadalajara, Mexico. DYNA, 86(209), 248254.

Graebel, W. (2007). Advanced fluid mechanics. Academic Press, USA.

Kondepudi, D., \& Prigogine, I. (2014). Modern thermodynamics: from heat engines to dissipative structures. John Wiley \& Sons, New York.

Leopold L., Wolman M., \& Miller J. (1964). Fluvial processes in geomorphology. San Francisco: Freeman

Leopold, B. (1953). Downstream change of velocity in rivers. American Journal of Science, 251(8), 606624.

Leopold, B., \& Langbein, W. (1962). The concept of entropy in landscape evolution. US Government Printing Office Washington, DC.

Seo, I., \& Cheong, T. (1998). Predicting longitudinal dispersion coefficient in natural streams. Journal of Hydraulic Engineering, 124(1), 25-32.

Varadhan, S. (2015). Entropy and its many Avatars. Journal of the Mathematical Society of Japan, 67(4), 1845-1857.

Zivieri, R., Pacini, N., Finocchio, G., \& Carpentieri, M. (2017). Rate of entropy model for irreversible processes in living systems. Scientific Reports, 7(1), 1-9. 